\title{
Study on Mechanical Effect of the Vehicle at Corners
}

\author{
Ming Ni \\ Department of Physics and Technology, Kunming University, Kunming 650214, China \\ kmxynm@163.com
}

Keywords: Accident; Sideslip; Dynamics; Rollover; Stability

\begin{abstract}
The experienced motorists know that they mustn't drive fast when they turn in the corners; especially in the rainy slippery situations, road accidents might occur. Vehicle rollover refers to a dangerous lateral movement of a vehicle in contact with the ground when it rotates around its longitudinal axis to a certain angle degree or greater angle during moving. There are many factors that could cause the vehicle's rollover, including automotive structures, road conditions and the driver's condition.
\end{abstract}

\section{Introduction}

In today's world, road traffic accidents are just like wars, diseases and natural disasters, which not only threaten people's lives and safety, but also cause huge economic losses to people's life, shrouding in a layer of dark shadow. In recent years, the car rollover accidents are increasing. Of all road traffic accidents, the car rollove is second only to the extent of the harm collision. Therefore, more and more experts and scholars study car stability. It is reported that since records that there have been motor vehicle road traffic fatalities began, the number of deaths from road accidents has reached up to more than 32 million. In the 20th century, the number of people serious injuries caused by road accidents each year and up to 500 million people hospitalized, the total number of injured people even was 300 million.

\section{Curve Speed Determines the Mechanical Effect}

With the increasing speed, the turning radius is increased The driver must increase the angle of the steering wheel to make the keep driving on the normal course of the bend. This bend radius increases with the speed of a phenomenon called the car "understeer"; However, it is just the opposite for the heavy-duty truck. That is, with the increasing speed, the vehicle "deviate"to the inside of the curve. This shows that the car turning radius is reduced with increasing speed.The driver must reduce the steering wheel angle to make the vehicle remain on the normal course of the bend. This radius increases with decreasing velocity curve phenomenon known as "oversteer". How to explain this phenomenon from the viewpoint of mechanics? It is based on the centrifugal force of inertia when the vehicle turns. When the vehicle moves at a high speed, the centrifugal force is so large that it maks the car skid outwards, producing a actual slight slip. For the front of the vehicle, the gravity center is close to its front wheels. During turning at a high speed of the vehicle, if the centrifugal force acting on the center of gravity in the front and rear wheels is divided into two force components, apparently the the force on front wheels is larger than that on the rear. This means that compared with the rear wheels, more static friction between the wheel and the ground will contend inertia centrifugal force. Thus the slipping outward of the front wheels will be greater than the rear ones, causing the deviation of vehicles moving towards the outer circumference motorsport. But it is opposite for overloaded trucks. Because its gravity center is close to the rear wheels. When the vehicle turns at a high speed, for the same reason, the the micro-slip rear wheels to the outside of is greater than the rear wheels, producing a circumferential inward deviation. 


\section{Skidding in the Flat Road When Turning}

Set the horizontal bend radius $\mathrm{R}$, the speed of the car when turninging is $\mathrm{v}$, then the size of the centripetal acceleration is

$$
a=\frac{v^{2}}{R}
$$

This acceleration is produced by the horizontal static friction $f_{s}$ caused by roads to the tires. According to Newton's Second Law, as $v$ increases, the centripetal acceleration increases rapidly (which is proportional to the square of the speed). Static friction will also increase accordingly. However, the increase of static friction is not unlimited. When the speed $\mathrm{v}$ reaches a certain value, the maximum static friction $f_{s \max }$, it can not be increased. At this point, if v continues to increase, it is insufficient to overcome the static friction sliding trend. So they spin the wheel to bend laterally outward from happening.

In the critical state when skidding begins:

$$
f_{s}=f_{s \max }=\mu_{s} m g=\frac{m v_{c}^{2}}{R}
$$

So

$$
v_{c}=\sqrt{\mu_{s} R g}
$$

Where $v_{c}$ is called the critical speed sideslip, $\mu_{s}$ is the static friction coefficient. Under normal circumstances, $\mu_{s}$ can not be found in the technical manual. It is usually obtained by the empirical formula. Their relationship is:

$$
\mu^{\prime}=0.97 \mu
$$

In the formula, $\mu^{\prime}$ is the horizontal sliding friction coefficient and $\mu$ is vertical sliding friction coefficient. Based on the above considerations, the practical level curve equation for critical speed sideslip is:

$$
v_{c}=\sqrt{\mu^{\prime} R g}
$$

\section{Corners Skidding and Rollover}

Vehicle rollover generally occurs in a large heavy-duty truck or a relatively high center of gravity of the vehicle, as shown in Figure 1, a truck is turning left, the height of whose center of gravity $C$ is $h$..

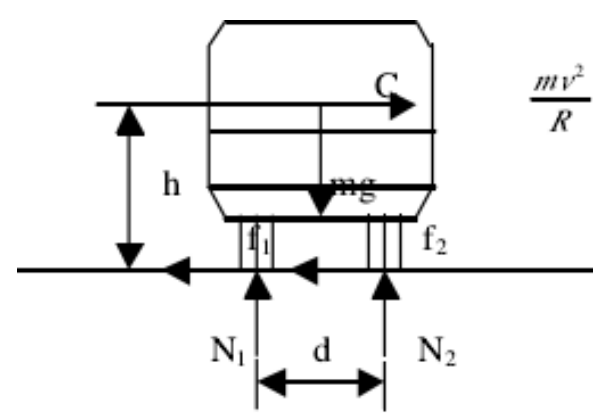

Figure. 1 the force diagram of vehical tipping

Take the truck as the reference for stress analysis. mg is the gravity acting on truck, $N_{1}$ and $N_{2}$ are the supportive forces by the ground of left and right wheels, $f_{1}$ and $f_{2}$ are the friction inside of the curve points, $m v^{2} / R$ is the function of the centrifugal force of inertia. Take the axis perpendicular to the paper as a shaft, Obtained by the torque balance:

$$
N_{1} d+\frac{m v^{2}}{R} h-m g \frac{d}{2}=0
$$


When $v=0$, namely in a stationary state, the above equation is transformed as

So

$$
N_{1} d-m g \frac{d}{2}=0
$$

$$
N_{1}=\frac{1}{2} m g
$$

That is: the load of the left and right wheels are equal, and each bears half the weight of the truck, However, it is different when the truck is turning. Obtained from the above equation:

$$
N_{1}=\frac{1}{2} m g-\frac{m v^{2} h}{R d}
$$

It shows that its load on the wheels reduces, and the reduced load is transferred to the outside of the wheels when the vehicle is turning. That is called "lateral load". With the increasing cornering speed $\mathrm{v} \quad N_{1}$ is decreased. In critical condition, $v=v_{c}$ and $N_{1}=0$. If the speed is equal to or greater than $v_{c}$, The vehicle rollover will occur. So the critical condition for the vehicle rollover is:

$$
m g \frac{d}{2}=\frac{m v^{2}}{R} h
$$

Solving above equation, it can be obtained as following

$$
v_{c}=\sqrt{\frac{g R d}{2 h}}
$$

Compare sliding formula $v_{c}=\sqrt{\mu^{\prime} R g}$ and rollover formula $v_{c}=\sqrt{g R d / 2 h}$ shows:

(1) If $\mu^{\prime}<d / 2 h$, then $v_{\mathrm{c}}$ turning $>v_{\mathrm{c}}$ sliding, The vehicle skidding occurs first, increasing the turning radius of the car after skidding. The critical velocity of rollover is greater. So the rollover of vehicles does not generally occur after skidding. The vehicle's center of gravity is low, and most meet the condition, $\mu^{\prime}<d / 2 h$. So skidding seldom occurs when cornering.

(2) If $\mu^{\prime}>d / 2 h$, then $v_{\text {cturning }}<v_{\text {csliding }}$. When the vehicle doesn't reach the slipping speed, and the speed has been reached or exceeded the rollover will occur first. The vehicle's center of gravity is high, which meet the above conditions. Generally rollover occurs first instead of skidding.

\section{Conclusions}

Vehicles move normally on the ground, which related to he driving force and the surface friction of the vehicles. The surface friction Hinders the sliding of the wheels. The wheels roll forward normally and at the same time bear the driving force provided by the vehicles on the ground. When the surface friction is too large and the driving force of the engine is too small ,underpower will show ,therefore accelerating will be weak. But the surface friction is too small and the driving force of the engine is too large. Once the driving force of the vehicle is greater than the maximum static friction force between the tire and the road surface, the car will skid. When the vehicle moves through the sharp turn, the rollover will occur if the speed is too fast, the cargo in the vehicle deviates the center of gravity, or lateral acceleration exceeds the limits. The skidding occurs on poor attached roads conditions. Sometimes the sideslip is exacerbated due to the slope of the road . If the sliding intensified lateral acceleration of the vehicle exceeds the limit and the vertical reaction force of the inside wheels is zero, the rollover will occur.

\section{References}

[1] Raymond M. Brach, R. Matthew Brach. A Review of Impact Models for Vehicle Collision. SAE Paper. No.870048. 1987:3-7

[2] Bruce Hongola, Cheng-Yao Chan. Simulation and Animation Tools for Analysis of Vehicle 
Collision: SMAC (Simulation Model Automobile Collisions)and Carmma (Simulation Animations). California PATH Working Paper. ISSN:1055-1417. 1999:3-5

[3] Cheng-Yao Chan. Studies of Vehicle Coll isions-A Documentation of the Simulation Codes: SMAC(Simulation Model of Automobile Collisions). California PATH Working Paper. ISSN 1055-1417. 1998:2-7

[4] Mchenry B. G., Mchenry R. R. SMAC97-refinement of the Collision -algorithm. SAE Paper. No.970947. 1997:1-3

[5] Dan A. Fittanto, Jordi Puig. Utilizing a Genetic Algorithm to Optimize Vehicle Simulation Trajectories: Determining Initial Velocity of a Vehicle in Yaw. SAE Paper. No.2000-01-1616. 2000:4-12

[6] Mchenry B.G., Mchenry R.R. CRASH 97-refinement of the Trajectory Solution Procedure. SAE Paper. No. 970949. 1997:2-4

[7] James A. Neptune, James E. Flynn. Impact Analysi s Based upon the CRASH Damage Algorithm. SAE paper. No. 950358. 1995:1-2

[8] Kenneth. Campbell.Energy Basis for Collision Severity. SAE Paper. No. 740565. 1974:1-4

[9] Mchenry. R. R, Mchenry. B. G. A. Revised Damage Analysis Procedure For The CRASH Computer Program. SAE Paper. No. 86 1894. 1986: l-2

[10] Marcus Hiemer, Sebastian Lehr, Uwe Kiencke. A Fuzzy System to Determine the Vehicle Yaw Angle. SAE Paper. No. 2004-01-191. 2004:1-9

[11] Strother C E, Woolley R L, James M B. A comparison between NHTSA crash test data and CRASH3 frontal stiffness coefficients[C]. SAE, 900101, 1990

[12] James A N, Crush stiffness coefficients, restitution constants, and a revision of Crash3 and SMC[C]. SAE 980029, 1998

[13] Hermann Steffan, B. C. Geile. A New Approach to Occupant Simulation through the Coupling of PC-Crash and MADYMO.SAE Paper. No. 1999-01-0444. 1999: 1-5

[14] Michael S. Varat, Stein E. Husher. Crash Pulse Modeling for Vehicle Safety Research. The 18“PESV Paper. No. 50 1. 200 1:1-5

[15] A. Moser, Steffan, G. Kasanieiey, The Pedestrian Model in PC-Crash the introduction of a Multi Body System and its Validation SAE 1999-01-1445

[16] Hermann Steffan, B. C. Geigl, Anoteas Moser, A. New Approach of Occupant Simulation through Coupling of PC-Crash and MADYMO.SAE 1999-01-0444 